\title{
Mobile thrombus extending from the distal segment of the left main coronary artery to the left anterior descending artery
}

Keywords: Mobile thrombus; Left main coronary artery disease

\section{Introduction}

Left main coronary artery disease was originally described by James Bryan Herrick, who also described the symptoms of myocardial infarction. Acute coronary syndromes characterized by thrombus formation in the left main coronary artery represent an uncommon form of atherosclerotic cardiac diseases. We describe a 77-year old female patient who had mobile thrombus extending from the distal segment of the left main coronary artery to the left anterior descending artery.

\section{Case Description}

A 77-year old female patient was found to have signs of acute lateral myocardial infarction (MI) in ECG recorded after a $3 \mathrm{~h}$ history of chest pain and an urgent percutaneous intervention was planned. Coronary angiography (CAG) showed an image consistent with thrombus formation extending from the distal segment of the left main coronary artery (LMCA) to the proximal part of the left anterior descending artery (LAD).

While images were being recorded from different angles, the thrombus was observed to proceed in distal direction after detachment. The mobile thrombotic segment moving distally resulted in impairment in coronary flow (Figure 1). Percutaneous coronary intervention/ stenting was deemed inappropriate, as the partial detachment of the thrombus originating from the left main coronary artery was associated with the obstruction of the LAD distal bed and subsequent impairment of LAD flow.

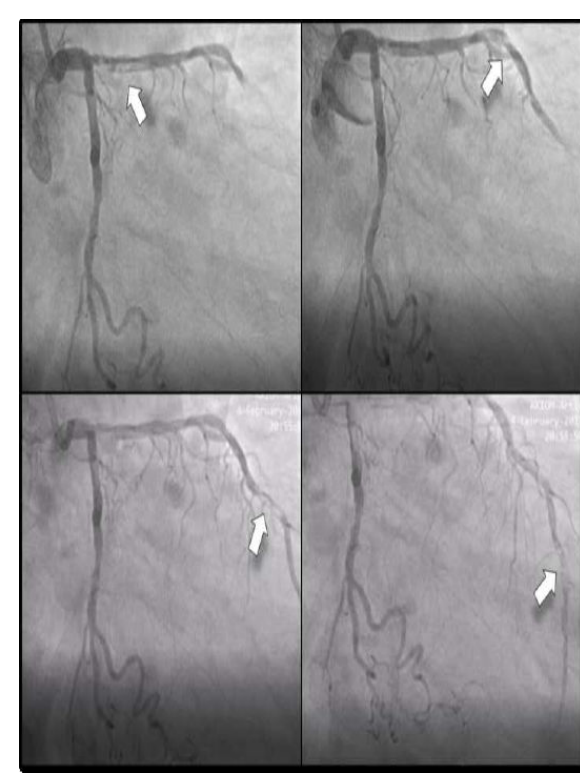

Ozcan Orscelik*, Bugra Ozkan, Ertan Emre Sahin, Veli Gokhan Cin, Mehmet Necdet Akkus and Tukay Ozcan

Department of Cardiology, Mersin University, Mersin/Turkey

*Author for correspondence:

ozcanorscelik@yahoo.com

Tel: +905304322474

Submitted: 01 June 2017

Accepted: 13 June 2017

Published online: 19 June 2017

Figure 1: Movement of detached part.

A consultation with the department of cardiovascular surgery was performed for an urgent coronary artery bypass grafting (CABG) procedure. Again, no potential benefit was expected from a CABG due to impaired distal LAD flow caused by the detached thrombotic segment. A follow-up CAG after thrombolytic infusion and intensive anti-aggregating therapy was scheduled. An intra-aortic aortic pump was placed and the patient was monitored in the coronary intensive care unit. On day 2 , the patient died after developing arrhythmic complications.

\section{Discussion}

Acute coronary thrombosis resulting in total occlusion in a coronary artery leads to myocardial infarction [1]. The underlying 
pathology in acute coronary syndromes involves the plaque rupture in coronary arteries that is associated with the synthesis of thrombogenic molecules and potential occlusion of the lumen of the coronary artery with platelet aggregates, fibrin, and red blood cells [2].

Left main coronary artery disease was originally described by James Bryan Herrick, who also described the symptoms of myocardial infarction [3]. Acute coronary syndromes characterized by thrombus formation in the left main coronary artery represent an uncommon form of atherosclerotic cardiac diseases [2]. Since this artery is responsible for the majority of the blood supply of the myocardium of the left ventricle, early diagnosis and urgent revascularization are essential [4]. Standard treatments include CABG, and percutaneous coronary intervention in some cases. It has been reported that in addition to thrombolytic treatment, antiplatelet agents may also be administered to patients with acute myocardial infarction $[5,6]$.

To our knowledge, although patients developing myocardial infarction due to thrombus formation in LMCA and/or LAD have been previously reported, no cases with a loose thrombus resulting in obstruction of the distal bed after partial detachment have been described. Therefore, whether these patients should be managed with percutaneous intervention, CABG, or thrombolytic therapy together with intensive antiaggregating treatment is not clearly known. Further information is warranted for such patients.

\section{Executive Summary}

Left main coronary artery disease was originally described by James Bryan Herrick, who also described the symptoms of myocardial infarction.

Acute coronary syndromes characterized by thrombus formation in the left main coronary artery represent an uncommon form of atherosclerotic cardiac diseases.

We describe a 77-year old female patient who had mobile thrombus extending from the distal segment of the left main coronary artery to the left anterior descending anterior artery.

\section{References}

1. DeWood MA, Spores J, Notske R, et al. Prevalence of total coronary occlusion during the early hours of transmural myocardial infarction. N. Engl. J. Med. 303: 897-902 (1980).

2. Neri R, Migliorini A, Moschi G, et al. Percutaneous reperfusion of left main coronary disease complicated by acute myocardial infarction. Catheter. Cardiovasc. Interv. 56: 31-34 (2002).

3. Herrick JB. Clinical features of sudden obstruction of the coronary arteries. JAMA. 250: 1757-1765 (1983).

4. Alipour-Parsa S, Farahani E. Successful Treatment of a Left Main Thrombus by Intracoronary Eptifibatide Infusion in a 36-Year-Old Patient. J. Tehran. Heart. Cent.11: 143-148 (2016).

5. Kleiman NS, Ohman EM, Califf RM, et al. Profound inhibition of platelet aggregation with monoclonal antibody 7E3 Fab after thrombolytic therapy. Results of the Thrombolysis and Angioplasty in Myocardial Infarction (TAMI) 8 Pilot Study. J. Am. Coll. Cardiol. 22: 381-389 (1993).

6. Ohman EM, Kleiman NS, Gacioch G, et al. Combined accelerated tissue-plasminogen activator and platelet glycoprotein IIb/IIIa integrin receptor blockade with
Integrilin in acute myocardial infarction. Results of a randomized, placebo-controlled, dose-ranging trial. IMPACTAMI Investigators. Circulation. 95: 846-854 (1997). 